\title{
The defense response in Arabidopsis thaliana against Fusarium sporotrichioides
}

Tomoya Asano ${ }^{1,2}$, Makoto Kimura ${ }^{3}$ and Takumi Nishiuchi ${ }^{1,4^{*}}$

\begin{abstract}
Background: Certain graminaceous plants such as Zea mays and Triticum aestivum serve as hosts for Fusarium sporotrichioides; however, molecular interactions between the host plants and F. sporotrichioides remain unknown. It is also not known whether any interaction between Arabidopsis thaliana and F. sporotrichioides can occur. To understand these interactions, we performed proteomic analysis.

Results: Arabidopsis leaves and flowers were inoculated with F. sporotrichioides. Accumulation of PLANT DEFENSIN1.2 (PDF1.2) and PATHOGENESIS RELATED1 (PR1) mRNA in Arabidopsis were increased by inoculation of F. sporotrichioides. Furthermore, mitogen-activated protein kinase 3 (MPK3) and mitogen-activated protein kinase 6 (MPK6), which represent MAP kinases in Arabidopsis, were activated by inoculation of F. sporotrichioides. Proteomic analysis revealed that some defense-related proteins were upregulated, while the expression of photosynthesis- and metabolism-related proteins was down regulated, by inoculation with F. sporotrichioides. We carried out the proteomic analysis about upregulated proteins by inoculation with Fusarium graminearum. The glutathione S-transferases (GSTs), such as GSTF4 and GSTF7 were upregulated, by inoculation with F. graminearuminfected Arabidopsis leaves. On the other hand, GSTF3 and GSTF9 were uniquely upregulated, by inoculation with F. sporotrichioides.
\end{abstract}

Conclusions: These results indicate that Arabidopsis is a host plant for F. sporotrichioides. We revealed that defense response of Arabidopsis is initiated by infection with F. sporotrichioides.

Keywords: Arabidopsis thaliana, Defense response, Fusarium sporotrichioides, T-2 toxin, MAP kinase, GSTs, Superoxide dismutase, Ascorbate peroxidase

\section{Background}

Fusarium head blight (FHB) is a severe disease that affects cereal crops worldwide [1,2]. This disease is caused by Fusarium species, such as Fusarium sporotrichioides, F. graminearum, and F. culmorum. The soilborne pathogen $F$. sporotrichioides is often observed in cold climates, such as in northern Japan, the northern USA, northern Europe, and Russia [3]. F. sporotrichioides was first isolated from corn in France and was identified as F. tricinctum NRRL 3229 [3]; it was later determined that this strain included both $F$. sporotrichioides and F. tricinctum. Similarly, the F. sporotrichioides IFO 9955

\footnotetext{
*Correspondence: tnish9@staff.kanazawa-u.ac.jp

${ }^{1}$ Division of Functional Genomics, Advanced Science Research Center,

Kanazawa University, 13-1 Takaramachi, Kanazawa 920-0934, Japan

${ }^{4}$ Division of Life Science, Graduate School of Natural Science and

Technology, Kanazawa University, Kanazawa 920-1192, Japan

Full list of author information is available at the end of the article
}

strain was identified from a bean hull by Ueno et al. [3], although it had previously been misidentified as F. solani [3]. It has been reported that overwintered cereals colonized by F. sporotrichioides caused the deaths of approximately 1,100 people in the erstwhile USSR during World War II [3].

Some Fusarium species also produce trichothecene mycotoxins, which are known to inhibit protein synthesis in eukaryotes [4,5]. Trichothecenes encompass many molecular species, which can be classified into 4 major groups (types A-D). Among these species, the type A (i.e., T-2 toxin) and type $B$ (i.e., deoxynivalenol [DON]) trichothecenes are distinguished by the absence or presence of a ketone group at the $\mathrm{C}-8$ position, respectively [6]. Trichothecenes are often found in cereal grains and cereal-derived commodities [6]. T-2 toxin has been reported to be approximately 10 times more toxic to mammals and plants than the type B trichothecenes,

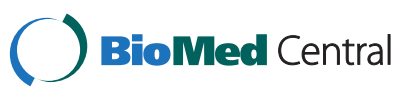


such as DON [7]. The tri5 mutant of F. graminearum, which cannot produce DON, can infect wheat florets and spikes, but it has decreased virulence against host plants [8]. Bai et al. suggested that DON supports the infection of $F$. graminearum in wheat [8]. On the other hand, the expression of PLANT DEFENSIN1.2 (PDF1.2) and PATHOGENESIS RELATED1 (PR1) mRNAs in Arabidopsis were induced by T-2 toxin [9]. Mitogen-activated protein kinase 3 (MPK3) and mitogen-activated protein kinase 6 (MPK6) in Arabidopsis have also been shown to be activated by T-2 toxin [9]. Nishiuchi et al. suggested that T-2 toxin possesses an elicitor-like activity [9].

The elicitors from Fusarium species induce the defense response of Arabidopsis cell suspension cultures [10,11]. The chitosan from $F$. moniliforme induced the expression of glutathione $S$-transferases (GSTs) in Arabidopsis, which is marker protein of pathogen defense response [11]. Moreover, some Fusarium species produce the mycotoxins, such as fumonisins [12]. The fumonisins are produced by $F$. moniliforme, [12] F. proliferatum, $F$. anthophilum, F. dlamini, F. napiforme and Alternaria alternata f. sp. lycopersici [12]. The fumonisins acts as an inhibitor of sphingosine $N$-acetyltransfearase [12], and the fumonisin B1 (FB1) causes the program cell death (PCD) in plants and animals [13]. Furthermore, the expression of $P R$ genes and PDF1.2 were induced by FB1 [13].

The upregulation of GSTs has been reported in type B-producing F. graminearum-infected wheat flowers [14]. GSTs function in the detoxification of both endogenous and xenobiotic compounds [15]. Gardiner et al. found evidence for nonenzymatic formation of DON-GSH conjugates in vitro using both liquid chromatography-mass spectrometry and nuclear magnetic resonance analysis [16] and suggested that GST is involved in DON detoxification [16]. Furthermore, disease symptoms manifested by Arabidopsis in response to F. graminearum are related to ethylene signaling [17]; other interactions between host plants and some Fusarium species have been reported. However, it is unknown whether there is an interaction between A. thaliana and F. sporotrichioides.

Proteomics is a tool to gain information on the protein levels, and it has frequently been used to study plant diseases $[18,19]$. Mukherjee et al. suggested that proteomic analysis of the defense response to Alternaria brassicicola can be compared to other types of plantpathogen interactions and leaf senescence in Arabidopsis [20]. The interaction of F. graminearum with some host plants has also previously been investigated by proteomic analysis. For instance, Triticum aestivum interacts with F. graminearum [21]. In this regard, Zhou et al. suggested that proteins related to jasmonic acid signaling pathways, PR protein, amino acid synthesis, and nitrogen metabolism were upregulated by inoculation of the plant with F. graminearum [21]. The defense response of Hordeum vulgare is also elicited by infection with F. graminearum [22]. Geddes et al. suggested that FHB caused increases in proteins associated with the oxidative burst and oxidative stress response, such as malate dehydrogenase, peroxidases, and PR protein [22]. However, although $F$. sporotrichioides produces the strong T-2 toxin, the molecular interaction between host plants and F. sporotrichioides is not understood.

In this paper, we report that Arabidopsis can act as a host to F. sporotrichioides. We demonstrate the defense response in Arabidopsis leaves caused by inoculation with F. sporotrichioides; furthermore, a proteomic analysis revealed induction of some defense response proteins by inoculation with $F$. sporotrichioides.

\section{Results and discussion}

In this study, we first examined the virulence of $F$. sporotrichioides in Arabidopsis rosette leaves and flower buds. Makandar et al. revealed that efficient infection and disease by F. graminearum occurs when the fungus is infiltrated into Arabidopsis leaves [23]. F. graminearum H3 and F. graminearum ZEA-1 strains [24] were reported to show the pathogenicity to cereals in a farm (unpublished data). F. graminearum H3 produces DON, and F. graminearum ZEA-1 produces zearalenone (ZEA) and DON [24]. We infiltrated with highly concentrated conidial suspensions $\left(1 \times 10^{5}\right.$ conidia/mL $)$ of plantfungal $F$. graminearum $\mathrm{H} 3$ or ZEA-1 into Arabidopsis leaves. The both infectious hyphae of $F$. graminearum ZEA-1 and F. graminearum $\mathrm{H} 3$ were observed at a rate of about $50 \%$ in inoculated Arabidopsis leaves at 2 days post inoculation (dpi). These results indicate that F. graminearum ZEA-1 and F. graminearum $\mathrm{H} 3$ are phytopathogens to Arabidopsis. Next, we inoculated the Arabidopsis leaves with highly concentrated conidial suspensions $\left(1 \times 10^{6}\right.$ conidia/mL $)$ of $F$. sporotrichioides (Figure 1). At $2 \mathrm{dpi}$, normal leaves were observed in about $72 \%$ of the Arabidopsis leaves that had been infiltrated with F. sporotrichioides (Figures 1B, F) compared with mock treatment leaves (Figures 1A, E). However, transparent regions were observed in about $21 \%$ of those leaves (Figures 1C, G), and infectious hyphae were observed in about $7 \%$ of those leaves (Figures 1D, H). On the other hand, hyphae of F. sporotrichioides were also observed in all the inoculated Arabidopsis flower buds (Figures 1O, P). Trypan blue staining showed that infectious hyphae were present in the leaves infiltrated with F. sporotrichioides at $2 \mathrm{dpi}$ (Figures $1 \mathrm{I}-\mathrm{N}$ ). Thus, these results indicate that Arabidopsis is a host plant of F. sporotrichioides.

We next examined whether T-2 toxin accumulated in F. sporotrichioides-inoculated leaves. The type B trichothecenes, such as DON, were detected by matrix- 


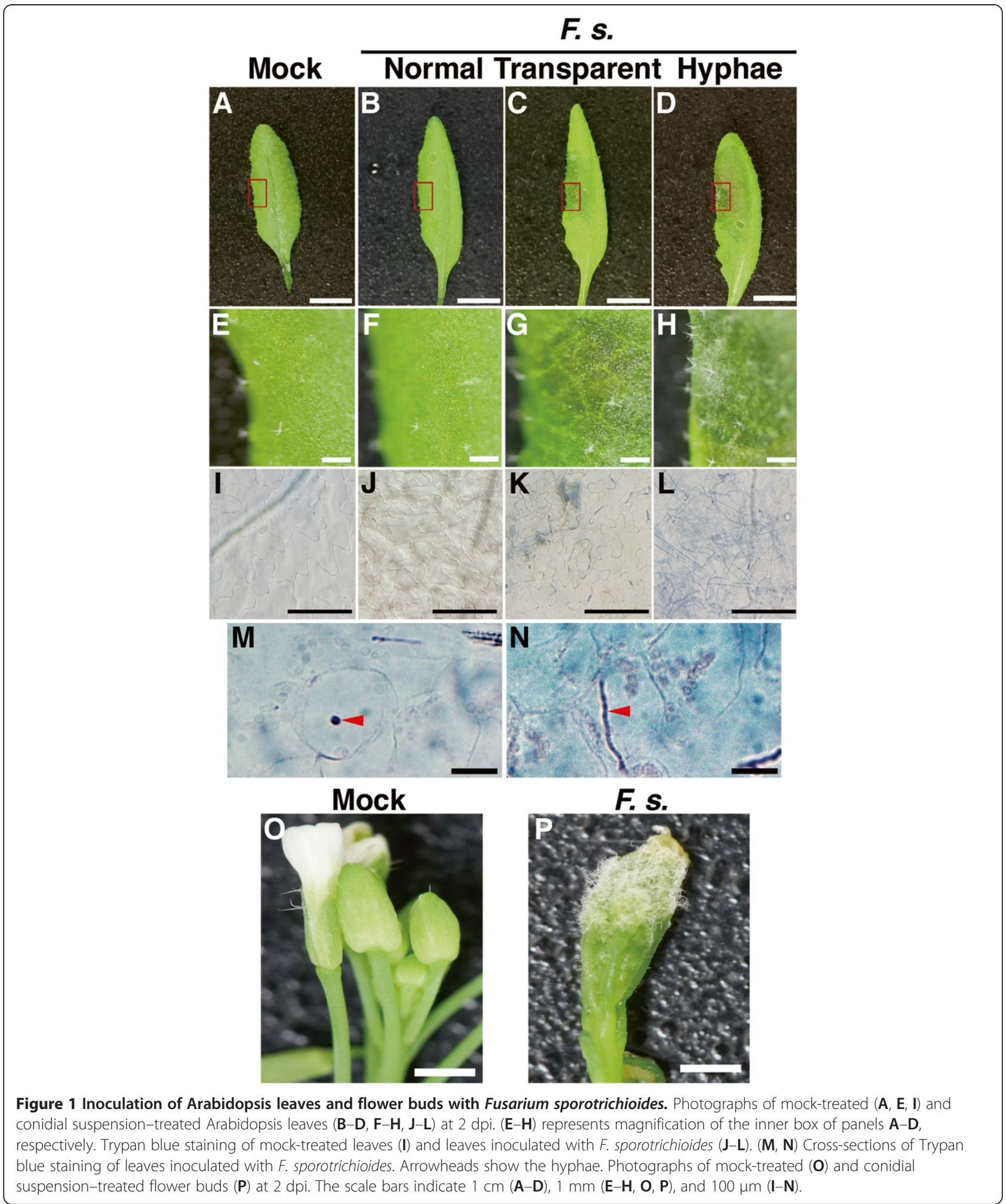

assisted laser desorption/ionization time-of-flight mass spectrometry (MALDI-TOF MS) [25]. Thus, we then established a method for quantification of $\mathrm{T}-2$ toxin using MALDI-TOF MS. We tested sodium azide as a matrix for detecting T-2 toxin. Additional file 1 shows that T-2 toxin was successfully ionized by sodium azide at $489.2 \mathrm{Da}$, corresponding to the sodium adduct of the toxin. In addition, the detection limit for T-2 toxin was 
approximately 50 fmol (data not shown). Another type A trichothecene, diacetoxyscirpenol (DAS), was also detected by the same method (Additional file 1). Because DAS was not detected in any of the samples inoculated with F. sporotrichioides, we used DAS as an internal control to quantify $\mathrm{T}-2$ toxin in F. sporotrichioidesinoculated tissues. We then measured the concentration of T-2 toxin in rosette leaves inoculated with conidial suspensions of $F$. sporotrichioides by the infiltration method. T-2 toxin appeared to have accumulated in the F. sporotrichioides-inoculated leaves to an average level of $17.6 \pm 6.84 \mathrm{ng}$ per leaf $(n=6)$, whereas T-2 toxin was not detected in the mock-treated leaves. The accumulation of this volume of T-2 toxin in Arabidopsis leaves caused the cell death and defense response [9]. Thus, the accumulation of T-2 toxin in the F. sporotrichioidesinoculated leaves was sufficient to contribute to their virulence.

After infiltration inoculation with highly concentrated microconidial suspensions, infectious F. sporotrichioides hyphae were observed in Arabidopsis leaf cells (Figures 1M, $\mathrm{N}$; arrowheads). Arabidopsis has previously been demonstrated to show interaction with $F$. sporotrichioides in that the expression of PR1 and PDF1.2a mRNAs in Arabidopsis were induced by inoculation with fumonisins-producing $F$. moniliforme [13], $F$. oxysporum [26], F. graminearum [9]. We therefore investigated the amount of PR1 and PDF1.2a mRNAs of F. sporotrichioides-inoculated Arabidopsis leaves by quantitative real-time RT-PCR (qRT-PCR) analysis. The PR1 mRNAs were induced by inoculation with $F$. sporotrichioides at 24 and $48 \mathrm{~h}$ post inoculation (Figure 2A). The PDF1.2a were induced by inoculation with F. sporotrichioides at $48 \mathrm{~h}$ post inoculation (Figure 2A).

Moreover, the activities of p47 (MPK6) and p44 (MPK3) have been shown to be activated by bacterial and fungal pathogen-associated molecular patterns (PAMPs) during plant-pathogen interactions [27]. MPK6 and MPK3 have also been shown to be activated by T-2 toxin and DON [9]. To investigate the defense response of Arabidopsis to F. sporotrichioides, we performed an in-gel kinase assay using myelin basic protein. The activities of MPK6 and MPK3 were not different between F. sporotrichioides-inoculated and mock-treated leaves by $6 \mathrm{~h}$ post inoculation (Figure 2B). However, the activities of MPK6 was increased by inoculation of F. sporotrichioides compared to mock treatment by $24 \mathrm{~h}$ post inoculation (Figure $2 \mathrm{~B}$ ). On the other hand, the activation of MPK3 was weak compared with MPK6 (Figure 2B). These results indicate that the defense response of Arabidopsis is induced by F. sporotrichioides infection.

To profile the defense response of Arabidopsis leaves against F. sporotrichioides, we performed a proteomic

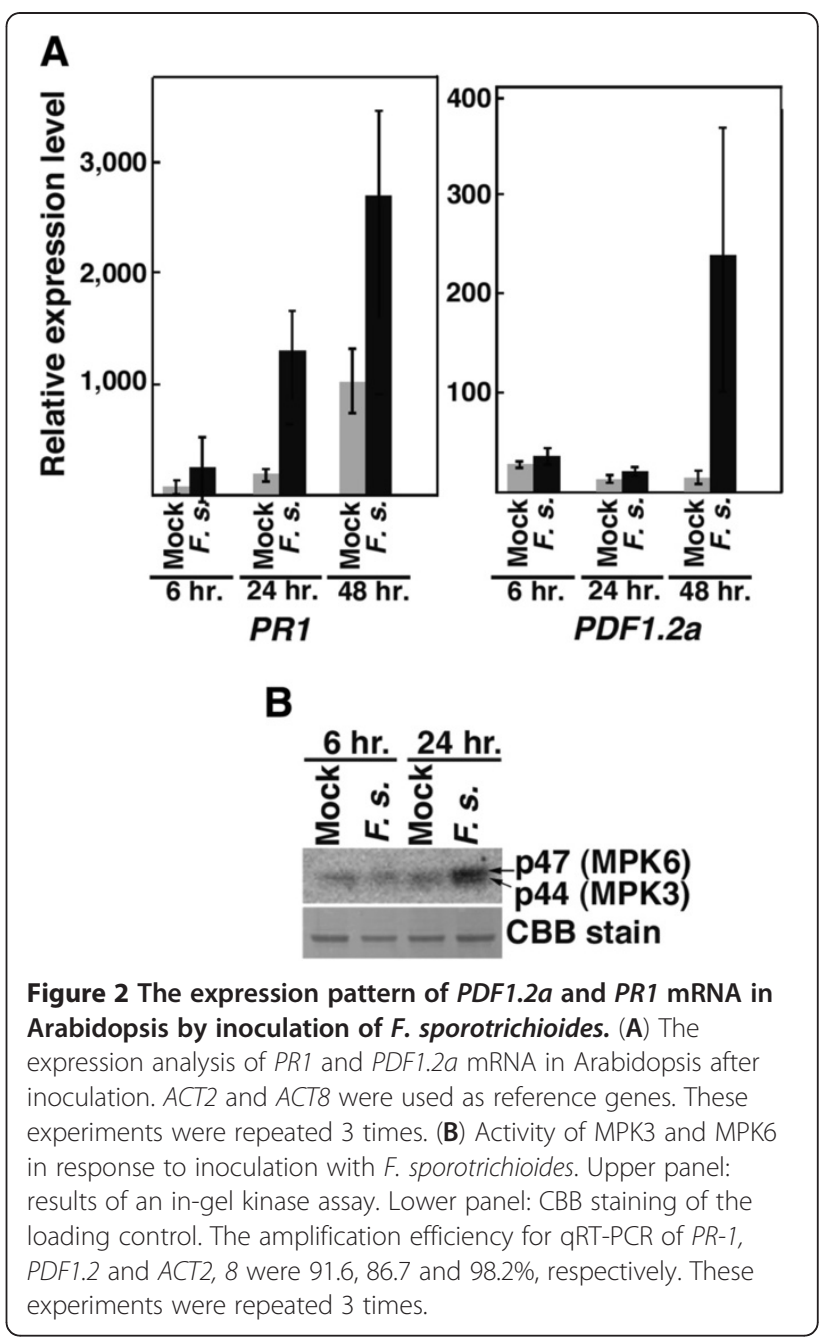

analysis in mature Arabidopsis leaves inoculated with F. sporotrichioides by the infiltration method. For this purpose, we performed two-dimensional (2D) difference gel electrophoresis. Total protein was extracted from mock-treated and F. sporotrichioides-inoculated Arabidopsis leaves, and fluorescently labeled with Cy3 and $\mathrm{Cy} 5$, respectively. The resulting fluorescently labeled proteins were mixed and subjected to $2 \mathrm{D}$ electrophoresis on the same gel. As shown in Figures 3A-E, 24 protein spots exhibited significantly different expression patterns (upregulated or down regulated) in Arabidopsis leaves inoculated with F. sporotrichioides. These protein spots were digested with trypsin, and the resulting peptides were identified by MALDI-TOF/TOF analyzer. Many proteins belonging to GSTs were increased in F. sporotrichioides-inoculated Arabidopsis leaves (Figure 3F). Table 1 shows that the levels of 5 GSTs from spots A3, A5, A7, A8, and A9 were upregulated to $2.66,3.28,1.82,204$, and 1.35 -fold by inoculation, respectively (Table 1 ). The spots A5 (GSTF4a) and A7 

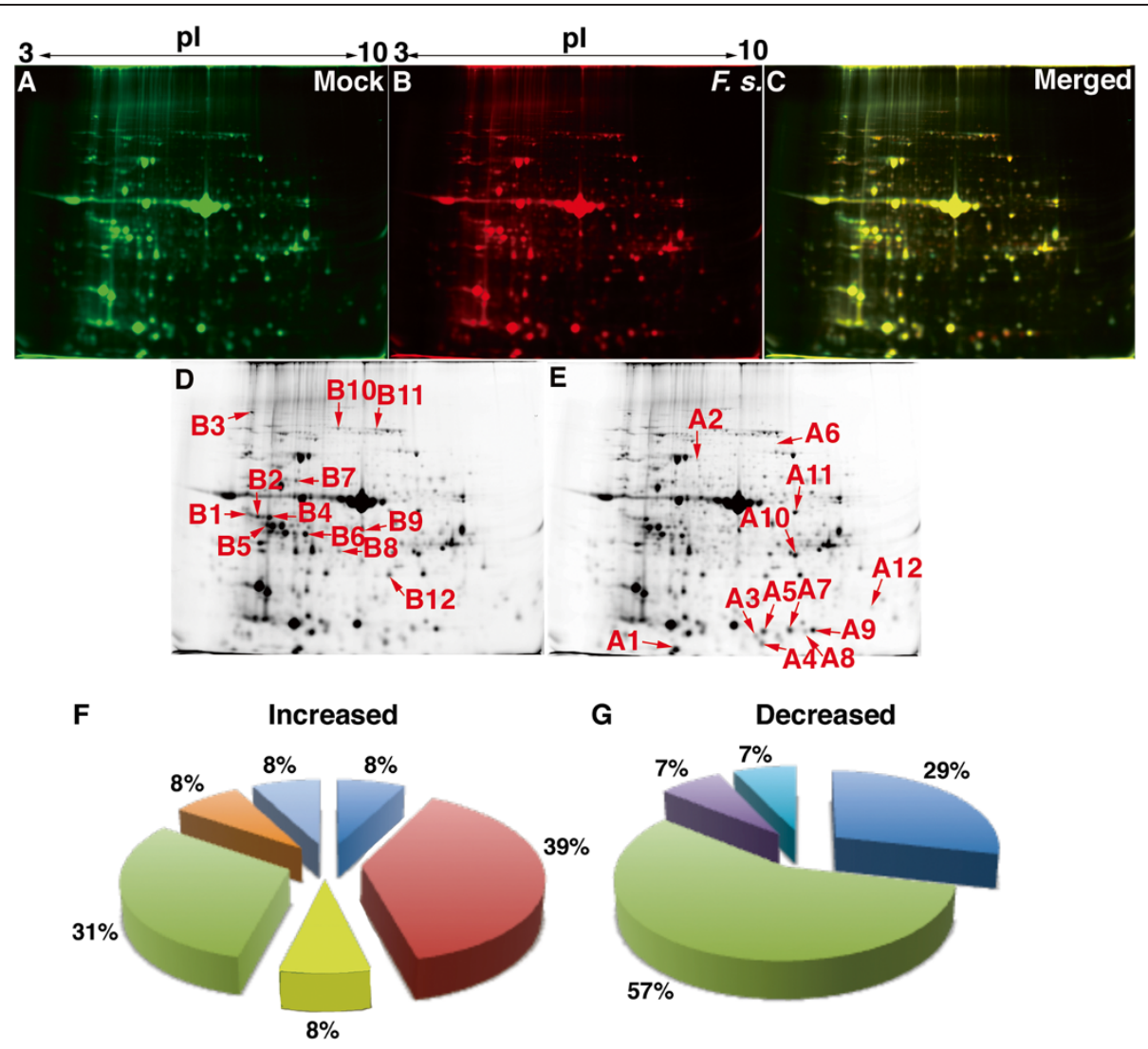

\section{Metabolism $\quad$ Photosynthesis $\square$ Protein synthesis $₫$ Miscellaneous aGTs Protein folding Redox Unknown}

Figure 3 Proteomic analysis of Arabidopsis leaves inoculated with a conidial suspension of $\boldsymbol{F}$. sporotrichioides. (A) Protein spots of mock-treated samples (Cy3-labeled total protein). (B) Protein spots of F. sporotrichioides-inoculated leaves (Cy5-labeled total protein). (C) Merge of $(\mathbf{A})$ and $(\mathbf{B})$ images. Spots of downregulated (D) and upregulated (E) proteins in F. sporotrichioides-inoculated leaves are shown. The spot numbers correspond to the results of protein identification (Table 1). This experiment was repeated 3 times. Functional classification of the identified proteins is shown. (F) The proteins increased and $(\mathbf{G})$ decreased in response to inoculation with F. sporotrichioides.

(GSTF4b) were identified as same GSTF4. Some posttranslational modifications of GSTs have been identified in Arabidopsis [28]. This different position of GSTF4 might be the post-translational modification. GSTs in Arabidopsis were used as marker protein of pathogen defense response [11]. GSTs are known to function in the maintenance of the redox state and the detoxification of toxins in many species [29]. The formation of DON-glutathione (DON-GSH) conjugates is likely to play a role in the detoxification of DON [16]. Therefore, these enhanced GSTs might be involved in the detoxification of T-2 toxin.

Moreover, superoxide dismutase (SOD) from spot A4 was upregulated to 1.3 -fold by inoculation (Table 1 ). The ascorbate peroxidase (APX) protein from spot A12 was enhanced to 2.0 -fold by inoculation (Table 1 ). It is known that T-2 toxin causes the accumulation of hydrogen peroxide in Arabidopsis leaves [9]. Accumulated reactive oxygen species (ROS) are detoxified by SOD and APX [30]. Thus, the induction of SOD and APX by inoculation of F. sporotrichioides might also be associated with the detoxification of superoxide in Arabidopsis leaves [29].

To compare the defense response in Arabidopsis to F. sporotrichioides and other Fusarium species, we carried out the proteomic analysis with inoculation of two known plant-fungal $F$. graminearum. F. graminearum $\mathrm{H} 3$ and ZEA-1 were used in this study. Total proteins of mock, F. graminearum $\mathrm{H} 3$ and F. graminearum ZEA-1-treated Arabidopsis leaves were fluorescently labeled with $\mathrm{Cy} 2, \mathrm{Cy} 3$ and $\mathrm{Cy} 5$, respectively. The expression of GSTF7 was upregulated to 1.20-fold by inoculation of F. graminearum $\mathrm{H} 3$ compared with mock treatment (Figure 4A, B, Table 2). GSTF4a, GSTF4b, GSTF3, GSTF9, APX and SOD were not increased with inoculation of F. graminearum H3 (Figure 4A, B, D, E, 
Table 1 Identification of protein spots that were differentially expressed after the inoculation of Arabidopsis leaves with $\boldsymbol{F}$. sporotrichioides

\begin{tabular}{|c|c|c|c|c|c|c|}
\hline Spot No. & Cov. $(\%)^{a}$ & Total $^{b}$ & Description & Species & Protein ID ${ }^{c}$ & Fusarium/Mock ${ }^{d}$ \\
\hline$\overline{\mathrm{A} 1}$ & 38.2 & 14.0 & LHCA1 & A. thaliana & gil15233115 & 6.15 \\
\hline A1 & 35.2 & 10.4 & Putative $\mathrm{H}^{+}$-transporting ATP synthase & A. thaliana & gi|18491181 & 6.15 \\
\hline A2 & 8.10 & 1.06 & Transketolase-like protein & A. thaliana & gi|7329685 & 62.4 \\
\hline A3 & 30.8 & 6.00 & Glutathione S-transferase 6 (GSTF3) & A. thaliana & gi|15218640 & 2.66 \\
\hline A4 & 29.5 & 12.0 & Superoxide dismutase & A. thaliana & gi|4455253 & 1.34 \\
\hline A5 & 59.9 & 18.8 & Glutathione S-transferase (GSTF4a) & A. thaliana & sp|P46422|GSTF4_ARATH & 3.28 \\
\hline A6 & 9.00 & 2.06 & Alpha-xylosidase & A. thaliana & sp|Q9S7Y7|XYL1_ARATH & 22.6 \\
\hline A7 & 57.5 & 15.9 & Glutathione S-transferase (GSTF4b) & A. thaliana & sp|P46422|GSTF4_ARATH & 1.82 \\
\hline A8 & 42.1 & 12.5 & Glutathione S-transferase 11 (GSTF9) & A. thaliana & sp|Q9SRY5|GSTF9_ARATH & 204 \\
\hline A9 & 54.4 & 24.0 & Glutathione S-transferase 9 (GSTF7) & A. thaliana & gi|15224581 & 1.35 \\
\hline A10 & 34.1 & 11.5 & ATP synthase gamma chain & A. thaliana & gi|5708095 & 1.15 \\
\hline A11 & 34.6 & 15.7 & Formate dehydrogenase & A. thaliana & gi|15241492 & 1.38 \\
\hline A12 & 12.5 & 3.20 & putative ascorbate Peroxidase APX4 & A. thaliana & gi|31980500 & 1.95 \\
\hline B1 & 44.4 & 18.4 & Rubisco activase & A. thaliana & gi|18405145 & 0.65 \\
\hline B2 & 44.3 & 24.4 & Rubisco activase & A. thaliana & gi|18405145 & 0.44 \\
\hline B3 & 10.4 & 11.9 & Embryo defective 2726 & A. thaliana & gi|18417320 & 0.57 \\
\hline B4 & 43.0 & 22.2 & Rubisco activase & A. thaliana & gi|18405145 & 0.76 \\
\hline B5 & 26.7 & 10.0 & Glutamine synthetase 2 & A. thaliana & gi|15238559 & 0.67 \\
\hline B6 & 38.5 & 20.0 & Rubisco activase & A. thaliana & gi|30687999 & 0.70 \\
\hline B7 & 17.1 & 13.1 & Chaperonin, putative & A. thaliana & gi|15231255 & 0.88 \\
\hline B7 & 18.3 & 6.01 & ATP synthase CF1 alpha subunit & A. thaliana & gi|7525018 & 0.88 \\
\hline B8 & 19.5 & 6.15 & $\begin{array}{l}\text { ATP synthase gamma chain, } \\
\text { chloroplast precursor }\end{array}$ & A. thaliana & gi|5708095 & 0.54 \\
\hline B8 & 7.80 & 4.00 & Indole-3-acetonitrile nitrilase & A. thaliana & gi|30692067 & 0.54 \\
\hline B9 & 12.9 & 6.09 & $\begin{array}{l}\text { Glyceraldehyde-3-phosphate } \\
\text { dehydrogenase B subunit }\end{array}$ & A. thaliana & gi|336390 & 0.96 \\
\hline B10 & 21.7 & 26.3 & $\begin{array}{l}\text { Arabidopsis thaliana glycine } \\
\text { Decarboxylase P-protein } 2\end{array}$ & A. thaliana & gi|15225249 & 0.78 \\
\hline B11 & 24.9 & 24.1 & $\begin{array}{l}\text { Arabidopsis thaliana glycine } \\
\text { Decarboxylase P-protein } 1\end{array}$ & A. thaliana & gi|15234036 & 0.65 \\
\hline B12 & 28.7 & 10.7 & Ferredoxin-NADPH(+)-Oxidoreductase 2 & A. thaliana & gi|15223753 & 0.89 \\
\hline
\end{tabular}

${ }^{a}$ Cov. (\%); Coverage: the percent ratio of all amino acids from valid peptide matches to the total number of amino acids in the protein. ${ }^{b}$ Total ProtScore was

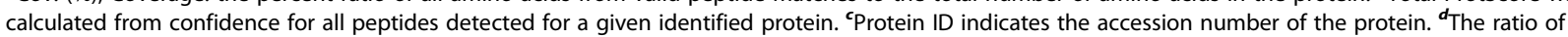
Fusarium/Mock shows the fold-change in expression levels between Fusarium-inoculated leaves and mock-treated leaves for each protein. Data are representative of 3 independent experiments.

Table 2). On the other hand, GSTF4a, GSTF4b, GSTF7 and APX were upregulated to 1.48, 1.19, 1.36 and 2.17-fold by inoculation of $F$. graminearum ZEA-1, respectively (Figure $4 \mathrm{~A}, \mathrm{C}, \mathrm{D}, \mathrm{F}$, Table 2). The extracellular matrix from $F$. moniliforme contains various elicitors to Arabidopsis, such as chitosan [11]. The expression of GSTF7 was enhanced by inoculation of F. sporotrichioides, F. graminearum $\mathrm{H} 3$ and F. graminearum ZEA-1 (Figure 4G). These results indicate that GSTF7 might be enhanced by common elicitors of Fusarium species. On the other hand, the expression of GSTF4a, GSTF4b and APX were increased with $F$. sporotrichioides and F. graminearum ZEA-1 (Figure 4G). We suggest that GSTF4a, GSTF4b, GSTF7 and APX were enhanced by common elicitors of $F$. sporotrichioides and F. graminearum $\mathrm{H} 3$.

Also, the expression of GSTF3, GSTF9 and SOD were uniquely enhanced by inoculation with $F$. sporotrichioides (Figure 4G). F. sporotrichioides produces trichothecenes, which is T-2 toxin [6]. T-2 toxin from F. sporotrichioides induces the defense response in Arabidopsis [9]. The expression of GSTF3, GSTF9 and SOD might be enhanced 


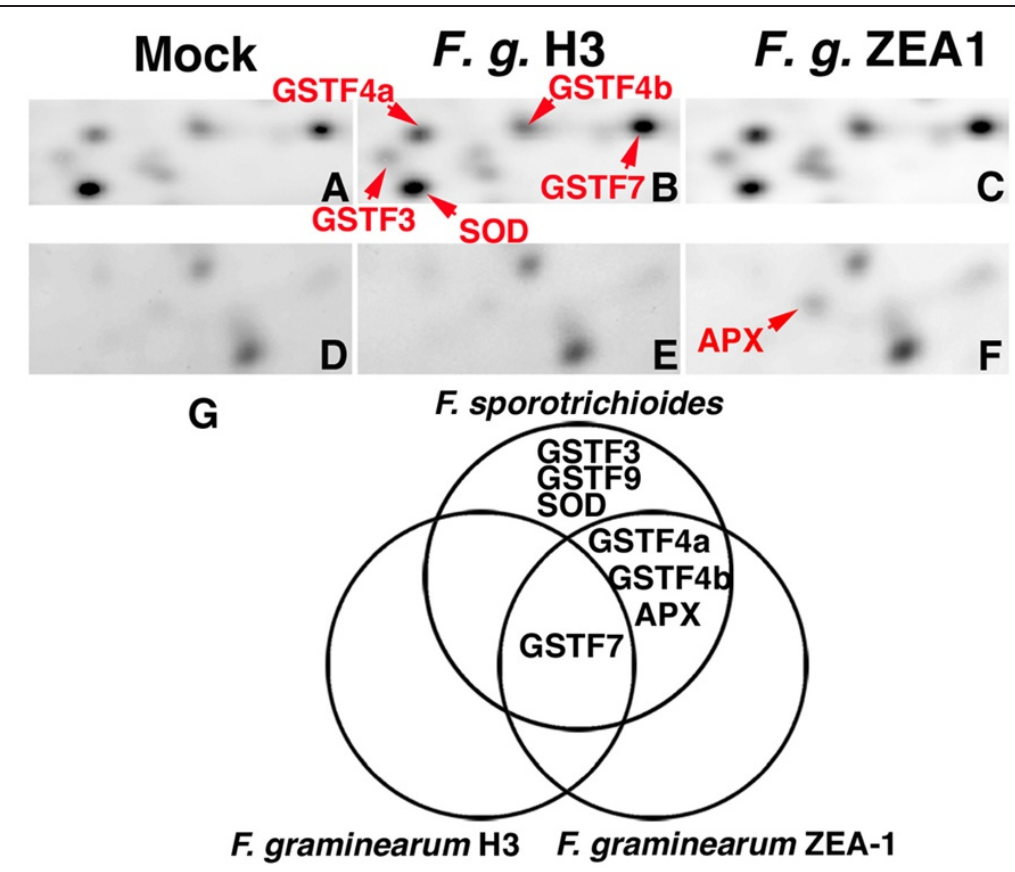

Figure 4 The expression pattern of GSTs, APX and SOD in Arabidopsis leaves inoculated with a conidial suspension of $F$. graminearum H3 and ZEA-1. (A, D) Protein spots of mock-treated samples (Cy2-labeled total protein). (B, E) Protein spots of F. graminearum H3-inoculated leaves (Cy3-labeled total protein). (C, F) Protein spots of F. graminearum ZEA-1-inoculated leaves (Cy5-labeled total protein). This experiment was repeated 3 times. (G) Venn diagram showing of expression pattern of GSTs, APX and SOD proteins in Arabidopsis leaves inoculated with a conidial suspension of F. sporotrichioides, F. graminearum $\mathrm{H} 3$ and ZEA-1.

by T-2 toxin. We suggest that the defense response in Arabidopsis against F. sporotrichioides causes by the various elicitors.

Conversely, the expression of photosynthesis- and metabolism-related proteins such as Rubisco activase, ATP synthase, and ferredoxin-NADP ${ }^{+}$oxidoreductase was downregulated in Arabidopsis leaves by inoculation with F. sporotrichioides (Figure 3G). Rubsico activase from spots B1, B2, B4, and B6 were downregulated $0.65,0.44,0.76$, and 0.70 -fold by inoculation (Table 1 ). Rubisco activase is required to allow the regeneration of the critical carbamate in the active site of Rubisco [31]. Moreover, glutamine synthetase 2 was decreased to 0.67 fold by inoculation (Table 1). Glutamine synthetase is one of the essential enzymes for glutamine production [32]. We propose that the activation of the defensesignaling pathway was enhanced, while the expression of photosynthesis- and metabolism-related proteins was suppressed by inoculation with F. sporotrichioides.

Five days after inoculation with $F$. graminearum, GSTs and SOD were upregulated or induced, and Rubisco activase were downregulated $[14,21]$. These results

Table 2 The protein spots that were differentially expressed after the inoculation of Arabidopsis leaves with two F. graminearum $\mathrm{H} 3$ and ZEA-1 strains

\begin{tabular}{|c|c|c|c|c|}
\hline Spot No. & Description & Protein $\mathrm{ID}^{a}$ & $\begin{array}{l}\text { F. graminearum } \\
\text { H3/Mock }^{b}\end{array}$ & $\begin{array}{l}\text { F. graminearum } \\
\text { ZEA-1/Mock }\end{array}$ \\
\hline A3 & Glutathione S-transferase 6 (GSTF3) & gi|15218640 & 1.03 & 1.13 \\
\hline A4 & Superoxide Dismutase & gi|4455253 & 1.10 & 1.10 \\
\hline A5 & Glutathione S-transferase (GSTF4a) & sp|P46422|GSTF4_ARATH & 1.13 & 1.48 \\
\hline A7 & Glutathione S-transferase (GSTF4b) & sp|P46422|GSTF4_ARATH & 1.03 & 1.19 \\
\hline A8 & Glutathione S-transferase 11 (GSTF9) & sp|Q9SRY5|GSTF9_ARATH & N. D. & N. D. \\
\hline A9 & Glutathione S-transferase 9 (GSTF7) & gi|15224581 & 1.20 & 1.36 \\
\hline $\mathrm{A} 12$ & Putative Ascorbate Peroxidase APX4 & gi|31980500 & 0.80 & 2.17 \\
\hline
\end{tabular}

${ }^{a}$ Protein ID indicates the accession number of the protein. ${ }^{\boldsymbol{b}}$ The ratio of Fusarium/Mock shows the fold-change in expression levels between Fusarium-inoculated leaves and mock-treated leaves for each protein. N. D. shows the not detected the spot. Data are representative of 3 independent experiments. 
indicate that the interaction between Arabidopsis and F. sporotrichioides is similar to the interaction between wheat and F. graminearum at the proteomic level.

\section{Conclusions}

Some phytopathogenic Fusarium species, including F. sporotrichioides, are known to produce type A trichothecenes, such as T-2 toxin. However, the interactions between type A trichothecene-producing Fusarium species and plants have not been well studied. In this study, virulence of $F$. sporotrichioides was observed in Arabidopsis leaves after inoculation. Even when the Arabidopsis leaves were infiltrated with conidial suspensions of $F$. sporotrichioides, invasive hyphae were observed in the leaves. PR1 and PDF1.2 mRNA were induced by inoculation of $F$. sporotrichioides. MPK3 and MPK6, which are MAP kinases in Arabidopsis, were also activated in $24 \mathrm{~h}$ by $F$. sporotrichioides. Proteomic analysis revealed that some defense-related proteins, including 5 GSTs, SOD, and APX, were increased in the $F$. sporotrichioides-infiltrated leaves. Also, GSTF3, GSTF9 and SOD were uniquely enhanced by inoculation of $F$. sporotrichioides. These results indicate that a defense response is caused in Arabidopsis leaves by infection with $F$. sporotrichioides.

\section{Methods}

\section{Plant and fungal growth}

The Columbia (Col-0) ecotype of A. thaliana (L.) Heynh. was used in this study. Arabidopsis seeds were sown in soil, placed at $4^{\circ} \mathrm{C}$ in the dark for 2 days, and subsequently grown at $22^{\circ} \mathrm{C}$ under a $16 / 8$-h light/dark cycle. F. sporotrichioides strain IFO 9955 (previously misidentified as Fusarium solani) was used in this study [33]. F. graminearum H3 (MAFF101551) and ZEA-1 strains were used in this study. The fungi was grown at $22^{\circ} \mathrm{C}$ under weak light on Synthetic Low Nutrient (SN) agar medium. The production of microconidia was induced by $\mathrm{SN}$ liquid medium $\left(0.1 \% \mathrm{KH}_{2} \mathrm{PO}_{4}, 0.1 \%\right.$ $\mathrm{KNO}_{3}, 0.1 \% \mathrm{MgSO}_{4} \cdot 7 \mathrm{H}_{2} \mathrm{O}, 0.05 \% \mathrm{KCl}, 0.02 \%$ glucose, $0.02 \%$ sucrose) [34].

\section{Fungal inoculation}

For the preparation of conidia, F. sporotrichioides was cultured in SN liquid medium with shaking for 2 days at $22^{\circ} \mathrm{C}$, in constant darkness. The conidia were collected by centrifugation $(14,000 \mathrm{~g}$ at room temperature for $5 \mathrm{~min}$ ) and were washed with phosphate-buffered saline (PBS) at least 3 times. The collected conidia were suspended in PBS and the number of conidia counted using a hemocytometer. For the infiltration inoculation, a conidial suspension $\left(1 \times 10^{6}\right.$ conidia/mL) or PBS (mock) without detergent was injected into the abaxial sides of the leaves with a needleless syringe [35]. The inoculated plants were incubated in a chamber under about $100 \%$ relative humidity, at $22^{\circ} \mathrm{C}$, and a $16 / 8$-h light/dark cycle.

\section{Trypan blue staining}

The hyphae of inoculated leaves were stained by previously described solution [36]. After staining, leaves were washed in chloral hydrate [36].

\section{The quantitative real-time RT-PCR}

Total RNA was isolated using the Plant RNA Isolation Mini Kit (Agilent Technologies, CA, USA). First-strand cDNA was synthesized using the PrimeScript RT Reagent Kit (Takara Bio, Shiga, Japan). The qRT-PCR was carried out with SYBR ${ }^{\circledR}$ Premix Ex Taq ${ }^{\text {Tix }}$ II (Perfect Real Time) (Takara Bio, Shiga, Japan), gene specific primer pairs for PR1, PDF1.2 or $A C T 2 / 8$, respectively, and cDNA as template. Arabidopsis $A C T 2 / 8$ was used as reference genes. The primers used for qRT-PCR were as previously described (PR1 [37], PDF1.2a [38], and ACT2/8 [39]). The qRT-PCR analysis was performed using the Mx3000P QPCR System (Agilent Technologies, CA, USA). The following PCR program was used: initial denaturation, $95^{\circ} \mathrm{C}, 10 \mathrm{~s} ; 40$ cycles of $95^{\circ} \mathrm{C}$ for $5 \mathrm{~s}, 60^{\circ} \mathrm{C}$ for $20 \mathrm{~s}$ and $72^{\circ} \mathrm{C}$ for $30 \mathrm{~s}$ with a temperature transition rate of $20^{\circ} \mathrm{C} / \mathrm{s}$; and a melting curve analysis, at $95^{\circ} \mathrm{C}$ for 0 $\mathrm{s}$ and $65^{\circ} \mathrm{C}$ for $15 \mathrm{~s}$, and an increase to $95^{\circ} \mathrm{C}$ with a temperature transition rate of $0.1^{\circ} \mathrm{C} / \mathrm{s}$. To generate a standard curve, homologous standards were used in all experiments. The cDNA quantities of target genes were calculated using Mxpro QPCR software (Agilent Technologies, CA, USA). The q-PCR analysis was carried out 3 times.

\section{In-gel kinase assay}

Crude extracts were prepared from $F$. sporotrichioidesinoculated or mock-treated leaves. An in-gel kinase assay was performed as previously described [9].

\section{Quantification of T-2 toxin}

The plant and fungal materials were ground to a fine powder in liquid nitrogen with a mortar and pestle. For T-2 toxin extraction, the fine powder was added to $10 \mathrm{~mL}$ of an acetonitrile-water (84:16) solution containing $1 \mu \mathrm{g} / \mathrm{mL}$ diacetoxyscirpenol (DAS) as an internal standard. The solution was then incubated on a rotator for $60 \mathrm{~min}$ at room temperature. The extraction mixture was centrifuged $(2,000 \mathrm{~g}$, at room temperature, for $5 \mathrm{~min})$, and the supernatant collected.

To purify T-2 toxin, we used MycoSep 227 Trich columns (Romer Labs, Inc., MO, USA). The extraction mixture was added to a test tube, and a column was slowly inserted into the test tube. The purified solutions (fraction 1) were collected using the columns and transferred to new tubes. An acetonitrile-water (84:16) 
solution $(3 \mathrm{~mL})$ containing $1 \mu \mathrm{g} / \mathrm{mL}$ DAS was added to the test tube, a column was slowly inserted into the test tube again, and these purified solutions were added to fraction 1. Aliquots of these extraction mixtures were evaporated using a SpeedVac concentrator.

To quantify T-2 toxin, the extracts were mixed with $1 \mathrm{mg} / \mathrm{mL}$ sodium azide as a matrix and analyzed using a 4800 Plus MALDI TOF/TOF ${ }^{\mathrm{TM}}$ analyzer (AB Sciex, CA, USA).

\section{Proteomic analysis of Arabidopsis rosette leaves inoculated with $F$. sporotrichioides}

The plant materials were ground to a fine powder in liquid nitrogen using a mortar and pestle. For protein extraction, approximately $2 \mathrm{~g}$ of this fine powder was added to $5 \mathrm{~mL}$ of PBS buffer containing 1\% Triton-X 100, $1 \mathrm{mM}$ phenylmethanesulfonyl fluoride (PMSF) and $1 / 1000$ protease inhibitor cocktail (Sigma-Aldrich, MO, USA) and the solution thoroughly mixed by vortexing. The extract was centrifuged $\left(14,000 \mathrm{~g}\right.$ at $4^{\circ} \mathrm{C}$ for $15 \mathrm{~min}$ ) and the supernatant collected. The concentration of protein in each sample was measured with an RC DC Protein Assay Kit (Bio-Rad Japan, Tokyo, Japan). Next, the protein was precipitated with $5 \%$ trichloroacetic acid (TCA), and the resulting pellet was washed with $100 \%$ acetone at least twice. The acetone was briefly evaporated from the protein by aspiration for $5 \mathrm{~min}$. The protein was subsequently dissolved in lysis buffer (8 M urea, 2\% CHAPS, and $30 \mathrm{mM}$ Tris- $\mathrm{HCl}, \mathrm{pH}$ 8.5). The insoluble material was removed by centrifugation $\left(15,000 \mathrm{~g}\right.$ at $4^{\circ} \mathrm{C}$ for $\left.15 \mathrm{~min}\right)$, and the $\mathrm{pH}$ of the supernatant (ranging from 8 to 9) confirmed with litmus paper.

The proteins were labeled using CyDye DIGE Fluors developed for fluorescence 2-D technology (GE Healthcare, Tokyo, Japan) according to the manufacturer's recommendations. Each sample was covalently labeled with a different fluorescent dye: $\mathrm{Cy} 3$ (mock) or Cy5 (Arabidopsis inoculated with $F$. sporotrichioides). CyDyelabeled proteins (each $30 \mu \mathrm{g}$ ) were loaded onto an 18-cm rehydration strip with an immobilized $\mathrm{pH}$ gradient of 3-10 and separated on a Multiphor electrophoresis unit (GE Healthcare, Tokyo, Japan) using the following setting: a 1.5 -h gradient from $300-3,500 \mathrm{~V}$, and $5 \mathrm{~h}$ at $3,500 \mathrm{~V}$. After the isoelectric focusing (IEF), the rehydration strip was mounted onto the top of a $10 \%$ SDS-polyacrylamide gel with a stacking gel, in a Hoefer SE 600 Ruby system (GE Healthcare, Tokyo, Japan). Electrophoresis was performed at a constant voltage of $10 \mathrm{~mA}$ for $7 \mathrm{~h}$. After the 2D electrophoresis, the acrylamide gels were directly scanned using a Typhoon ${ }^{\mathrm{TM}}$ 9400 imager (GE Healthcare, Tokyo, Japan). The scanned images were applied to ImageQuant V5.2 software (GE Healthcare, Tokyo, Japan). PDQuest Advanced software
(Bio-Rad Japan, Tokyo, Japan) was used to compare individual images and to localize some of the spots. Differentially expressed proteins were identified by Student's $t$-test of spot intensity $(\mathrm{P}<0.05, \mathrm{n}=3)$. The protein spots were collected from the gels using an Ettan Spot Picker (GE Healthcare, Tokyo, Japan).

\section{Identification of proteins by MALDI-TOF/TOF analysis} MALDI-TOF/TOF analysis was performed, as previously described [40].

\section{Functional classification of proteins}

The identified proteins were classified according to functional categories using The Plant Proteome Database (http://ppdb.tc.cornell.edu/default.aspx).

\section{Additional file}

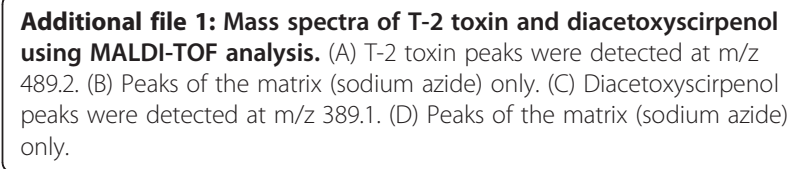

\section{Competing interests}

The authors declare that they have no competing interests.

\section{Authors' contributions}

TA carried out the experiments. MK isolated F. graminearum $\mathrm{H} 3$ and ZEA-1 strains. TA and TN designed experiments and wrote the manuscript. TN designed this project. All authors read and approved the final manuscript.

\section{Acknowledgements}

We are grateful to Prof. Kim Hammond-Kosack (Rothamsted Research, UK) for her very valuable suggestions and to Dr. Takayuki Aoki (RIKEN, Japan) for helpful discussions. This work was supported by Grants-in-Aid for Young Scientists (no. 21770038) and Scientific Research (no. 23580060) from the Ministry of Education, Culture, Sports, Science and Technology of Japan. The work was also supported in part by Research for Promoting Technological Seeds (no. 07-070) from the Japan Science and Technology Agency (JST).

\section{Author details}

${ }^{1}$ Division of Functional Genomics, Advanced Science Research Center, Kanazawa University, 13-1 Takaramachi, Kanazawa 920-0934, Japan. ${ }^{2}$ Equipment Support Promotion office, Advanced Science Research Center, Kanazawa University, Kanazawa 920-1192, Japan. ${ }^{3}$ Plant \& Microbial Metabolic Engineering Research Unit, Discovery Research Institute (DRI), RIKEN, 2-1 Hirosawa, Wako, Saitama 351-0198, Japan. ${ }^{4}$ Division of Life Science, Graduate School of Natural Science and Technology, Kanazawa University, Kanazawa 920-1192, Japan.

Received: 29 July 2012 Accepted: 5 October 2012 Published: 30 October 2012

\section{References}

1. Walter S, Nicholson P, Doohan FM: Action and reaction of host and pathogen during Fusarium head blight disease. New Phytol 2010, 185:54-66.

2. $X u X M$, Nicholson $P$, Ritieni A: Effects of fungal interactions among Fusarium head blight pathogens on disease development and mycotoxin accumulation. Int J Food Microbiol 2007, 119:67-71.

3. Marasas WF, Nelson PE, Toussoun TA: Toxigenic Fusarium species, identity and mycotoxicology. University Park: Pennsylvania State University Press; 1984.

4. McCormick SP, Stanley AM, Stover NA, Alexander NJ: Trichothecenes: from simple to complex mycotoxins. Toxins (Basel) 2011, 3:802-814. 
5. Li Y, Wang Z, Beier RC, Shen J, De Smet D, De Saeger S, Zhang S: T-2 toxin, a trichothecene mycotoxin: review of toxicity, metabolism, and analytical methods. J Agric Food Chem 2011, 59:3441-3453.

6. Mirocha CJ, Xie W, Filho ER: Chemistry and detection of Fusarium mycotoxins. In Fusarium head blight of wheat and barley. Edited by Leonard KJ, Bushnell WR. Saint Paul: American Phytopathological Society Press; 2003:144-164.

7. Ueno Y: Trichothecenes. In Chemical, biological and toxicological aspects. Edited by Ueno Y. Amsterdam: Elsevier; 1983:135-146.

8. Bai $\mathrm{GH}$, Desjardins AE, Plattner RD: Deoxynivalenol-nonproducing Fusarium graminearum causes initial infection, but does not cause disease spread in wheat spikes. Mycopathologia 2002, 153:91-98.

9. Nishiuchi T, Masuda D, Nakashita H, Ichimura K, Shinozaki K, Yoshida S, Kimura M, Yamaguchi I, Yamaguchi K: Fusarium phytotoxin trichothecenes have an elicitor-like activity in Arabidopsis thaliana, but the activity differed significantly among their molecular species. Mol Plant Microbe Interact 2006, 19:512-520.

10. Chivasa S, Hamilton JM, Pringle RS, Ndimba BK, Simon WJ, Lindsey K, Slabas AR: Proteomic analysis of differentially expressed proteins in fungal elicitor-treated Arabidopsis cell cultures. J Exp Bot 2006, 57:1553-1562.

11. Ndimba BK, Chivasa S, Hamilton JM, Simon WJ, Slabas AR: Proteomic analysis of changes in the extracellular matrix of Arabidopsis cell suspension cultures induced by fungal elicitors. Proteomics 2003, 3:1047-1059.

12. Sweeney MJ, Dobson AD: Molecular biology of mycotoxin biosynthesis. FEMS Microbiol Lett 1999, 175:149-163.

13. Stone JM, Heard JE, Asai T, Ausubel FM: Simulation of fungal-mediated cell death by fumonisin B1 and selection of fumonisin B1-resistant ( $f b r)$ Arabidopsis mutants. Plant Cell 2000, 12:1811-1822.

14. Zhou W, Kolb FL, Riechers DE: Identification of proteins induced or upregulated by Fusarium head blight infection in the spikes of hexaploid wheat (Triticum aestivum). Genome 2005, 48:770-780.

15. Marrs KA: The Functions and Regulation of Glutathione S-Transferases in Plants. Annu Rev Plant Physiol Plant Mol Biol 1996, 47:127-158.

16. Gardiner SA, Boddu J, Berthiller F, Hametner C, Stupar RM, Adam G, Muehlbauer GJ: Transcriptome analysis of the barley-deoxynivalenol interaction: evidence for a role of glutathione in deoxynivalenol detoxification. Mol Plant Microbe Interact 2010, 23:962-976.

17. Chen X, Steed A, Travella S, Keller B, Nicholson P: Fusarium graminearum exploits ethylene signalling to colonize dicotyledonous and monocotyledonous plants. New Phytol 2009, 182:975-983.

18. Gonzalez-Fernandez R, Jorrin-Novo JV: Contribution of proteomics to the study of plant pathogenic fungi. J Proteome Res 2012, 11:3-16.

19. Gonzalez-Fernandez R, Prats E, Jorrin-Novo JV: Proteomics of plant pathogenic fungi. J Biomed Biotechnol 2010, 2010:932527.

20. Mukherjee AK, Carp MJ, Zuchman R, Ziv T, Horwitz BA, Gepstein S: Proteomics of the response of Arabidopsis thaliana to infection with Alternaria brassicicola. J Proteomics 2010, 73:709-720.

21. Zhou W, Eudes F, Laroche A: Identification of differentially regulated proteins in response to a compatible interaction between the pathogen Fusarium graminearum and its host, Triticum aestivum. Proteomics 2006, 6:4599-4609.

22. Geddes J, Eudes F, Laroche A, Selinger LB: Differential expression of proteins in response to the interaction between the pathogen Fusarium graminearum and its host, Hordeum vulgare. Proteomics 2008, 8:545-554.

23. Makandar R, Nalam V, Chaturvedi R, Jeannotte R, Sparks AA, Shah J: Involvement of salicylate and jasmonate signaling pathways in Arabidopsis interaction with Fusarium graminearum. Mol Plant Microbe Interact 2010, 23:861-870

24. Igawa T, Takahashi-Ando N, Ochiai N, Ohsato S, Shimizu T, Kudo T, Yamaguchi I, Kimura M: Reduced contamination by the Fusarium mycotoxin zearalenone in maize kernels through genetic modification with a detoxification gene. Appl Environ Microb 2007, 73:1622-1629.

25. Blechova P, Havlova P, Gajdosova D, Havel J: New possibilities of matrixassisted laser desorption ionization time of flight mass spectrometry to analyze barley malt quality. Highly sensitive detection of mycotoxins. Environ Toxicol 2006, 21:403-408.

26. Epple $\mathrm{P}$, Apel $\mathrm{K}$, Bohlmann $\mathrm{H}$ : Overexpression of an endogenous thionin enhances resistance of Arabidopsis against Fusarium oxysporum. Plant Cell 1997, 9:509-520
27. Samajova O, Plihal O, Al-Yousif M, Hirt H, Samaj J: Improvement of stress tolerance in plants by genetic manipulation of mitogen-activated protein kinases. Biotechnol Adv 2011, In press.

28. Jones AM, Thomas V, Truman B, Lilley K, Mansfield J, Grant M: Specific changes in the Arabidopsis proteome in response to bacterial challenge: differentiating basal and $R$-gene mediated resistance. Phytochemistry 2004, 65:1805-1816.

29. Foyer $\mathrm{CH}$, Noctor $\mathrm{G}$ : Ascorbate and glutathione: the heart of the redox hub. Plant Physiol 2011, 155:2-18

30. Vanacker $\mathrm{H}$, Carver $\mathrm{TL}$, Foyer $\mathrm{CH}$ : Pathogen-induced changes in the antioxidant status of the apoplast in barley leaves. Plant Physiol 1998, 117:1103-1114.

31. Portis AR Jr, Li C, Wang D, Salvucci ME: Regulation of Rubisco activase and its interaction with Rubisco. J Exp Bot 2008, 59:1597-1604.

32. Lillo C: Signalling cascades integrating light-enhanced nitrate metabolism. Biochem J 2008, 415:11-19.

33. Ishii $\mathrm{K}$, Ueno $\mathrm{Y}$ : Isolation and characterization of two new trichothecenes from Fusarium sporotrichioides strain M-1-1. Appl Environ Microbiol 1981, 42:541-543.

34. Urban M, Daniels S, Mott E, Hammond-Kosack K: Arabidopsis is susceptible to the cereal ear blight fungal pathogens Fusarium graminearum and Fusarium culmorum. Plant Journal 2002, 32:961-973.

35. Makandar R, Essig JS, Schapaugh MA, Trick HN, Shah J: Genetically engineered resistance to Fusarium head blight in wheat by expression of Arabidopsis NPR1. Mol Plant Microbe In 2006, 19:123-129.

36. Mackey D, Belkhadir Y, Alonso JM, Ecker JR, Dangl JL: Arabidopsis RIN4 is a target of the type III virulence effector AvrRpt2 and modulates RPS2mediated resistance. Cell 2003, 112:379-389.

37. Hossain MM, Sultana F, Kubota M, Koyama H, Hyakumachi M: The plant growth-promoting fungus Penicillium simplicissimum GP17-2 induces resistance in Arabidopsis thaliana by activation of multiple defense signals. Plant Cell Physiol 2007, 48:1724-1736.

38. Clarke SM, Cristescu SM, Miersch O, Harren FJ, Wasternack C, Mur LA Jasmonates act with salicylic acid to confer basal thermotolerance in Arabidopsis thaliana. New Phytol 2009, 182:175-187.

39. Asano T, Masuda D, Yasuda M, Nakashita H, Kudo T, Kimura M, Yamaguchi K, Nishiuchi T: AtNFXL1, an Arabidopsis homologue of the human transcription factor NF-X1, functions as a negative regulator of the trichothecene phytotoxin-induced defense response. Plant Journal 2008, 53:450-464

40. Asano T, Nishiuchi T: Comparative Analysis of Phosphoprotein Expression Using 2D-DIGE. Methods Mol Biol 2011, 744:225-233.

doi:10.1186/1477-5956-10-61

Cite this article as: Asano et al.: The defense response in Arabidopsis thaliana against Fusarium sporotrichioides. Proteome Science 2012 10:61.

\section{Submit your next manuscript to BioMed Central and take full advantage of:}

- Convenient online submission

- Thorough peer review

- No space constraints or color figure charges

- Immediate publication on acceptance

- Inclusion in PubMed, CAS, Scopus and Google Scholar

- Research which is freely available for redistribution 